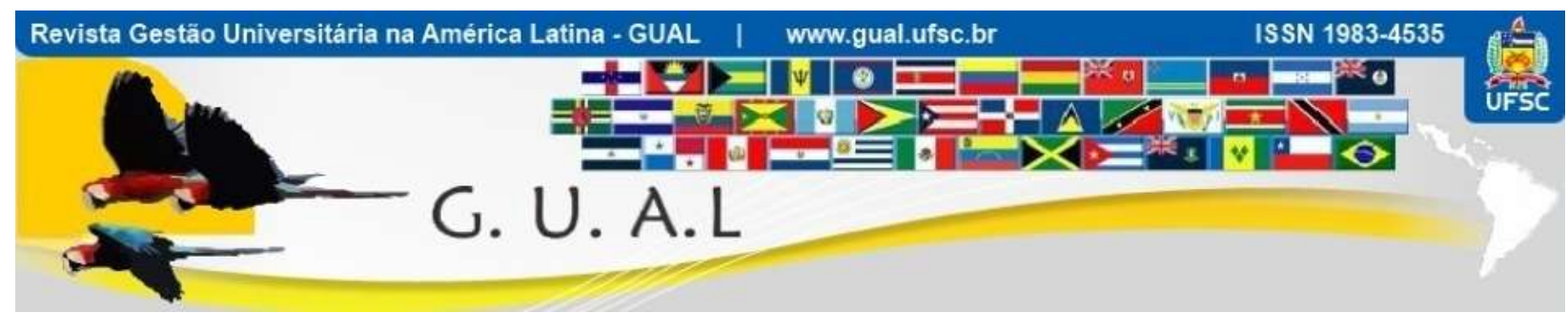

DOI: https://doi.org/10.5007/1983-4535.2021.e76356

\title{
QUALIDADE PERCEBIDA POR ALUNOS DE INSTITUIÇÕES DE ENSINO SUPERIOR: EFEITOS DO TAMANHO DA TURMA, GÊNERO E TEMPO DE EXPERIENNCIA DO ALUNO
}

\section{PERCEIVED QUALITY BY HIGHER EDUCATION STUDENTS: EFFECTS FROM CLASS SIZE, GENDER AND EXPERIENCE OF USE}

Marcus Rodrigues Peixoto, Mestre http://orcid.org/0000-0001-5131-8965 marcuspeixoto2002@hotmail.com Pontifícia Universidade Católica | Programa de Pós-Graduação em Administração Rio de Janeiro | Rio de Janeiro | Brasil

Jorge Brantes Ferreira, Doutor http://orcid.org/0000-0002-9579-4477 jorge.brantes@gmail.com Pontifícia Universidade Católica | Programa de Pós-Graduação em Administração Rio de Janeiro | Rio de Janeiro | Brasil

Recebido em 11/agosto/2020

Aprovado em 24/fevereiro/2021

Publicado em 03/março/2021

Sistema de Avaliação: Double Blind Review 


\title{
RESUMO
}

Objetiva-se investigar relações entre qualidade percebida no serviço educacional dos alunos de graduação presencial privada com: tamanho da turma, gênero e tempo de experiência de uso do serviço. Realizou-se pesquisa do tipo survey, de natureza quantitativa e caráter descritivo. A escala SERVPERF foi adaptada para mensurar as variáveis propostas sobre amostra de 1.379 estudantes de três instituições de ensino superior (IES) privadas brasileiras. Resultados do teste Mann-Whitney $U$ indicaram relações negativas significativas entre qualidade percebida e tamanho da turma. As percepções de qualidade dos alunos veteranos também apresentaram resultados significativamente inferiores às percepções dos calouros. Por outro lado, apesar das médias do grupo feminino serem superiores às do grupo masculino em todas dimensões pesquisadas, não foi encontrado resultado significativo entre qualidade percebida e gênero, de forma geral. Devido às lacunas em pesquisas com as variáveis avaliadas, os achados contribuem para debate acadêmico, sobretudo acerca do comportamento do consumidor. Adicionalmente, discutem-se sugestões relevantes para tomadas de decisões gerenciais das IES que buscam melhorias operacionais e financeiras e se encontram pressionadas por tendências mercadológicas, incluindo aumento de alunos por sala de aula e consequente risco de deterioração da qualidade percebida de seus serviços.

Palavras-chave: Qualidade Percebida. Experiência de Uso. Gênero. SERVPERF. Tamanho da Turma.

\begin{abstract}
This study intends to investigate the relationships between perceived quality in private Higher Education and class size, gender, and experience of use (i.e.: service consumption duration). Following a quantitative approach, a survey was employed to gather data from a sample of 1,379 students from three large Brazilian Higher Education Institutions (HEI). Results from a Mann-Whitney's U test indicate a significant negative relationship between perceived quality and class size. Additionally, senior students' perceived quality is significantly lower than firstyear students' perceptions of service quality. On the other hand, although female's average perceptions showed higher results than male's in all quality dimensions, no significance between perceived quality and gender was detected. The study highlights the potential impact of relevant items on HEI's service perceived quality and consumer behavior debate by covering current academic gaps on some of the variables' relationships analyses. HEIs can use these findings to improve their future strategic decisions, including valuable inputs to face current and future challenges, such as the need for operational and financial improvements and the management of the complex balance between the class size trends and service's perceived quality.
\end{abstract}

Keywords: Perceived Quality. Service Consumption Duration. Gender. SERVPERF. Class Size. 


\section{QUALIDADE PERCEBIDA POR ALUNOS DE INSTITUIÇÕES DE ENSINO SUPERIOR: EFEITOS DO TAMANHO \\ DA TURMA, GÊNERO E TEMPO DE EXPERIÊNCIA DO ALUNO \\ DOI: https://doi.org/10.5007/1983-4535.2021.e76356}

\section{INTRODUÇÃO}

O cenário econômico adverso e contínuo arrefecimento da competição são desafios que vêm impactando significativamente os negócios das Instituições de Ensino Superior (IES) brasileiras. Dados do INEP (2019) demonstram que as IES sofreram redução no total de matrículas presenciais de aproximadamente 7\% entre 2015 e 2018 e enfrentam problemas em suas receitas. Por exemplo, observa-se, no caso de IES privadas, queda no valor médio das mensalidades no mesmo período de $6 \%$ no ensino presencial e de $20 \%$ no EaD, além de piora no mix de receitas, com crescimento da participação do $\mathrm{EaD}$, modalidade de mensalidade inferior, com 30\% do total de alunos em 2018 versus 21\% em 2015 (HOPER, 2018).

O setor de educação superior privado, tanto no Brasil quanto no mundo, é impactado por crescentes níveis de competição interna e pressões mercadológicas, como maior competição global e diminuição orçamentária, e pela busca de vantagens competitivas em um ambiente concorrencial ainda bastante pulverizado (PEDRO; MENDES; LOURENÇO, 2018; LIM; JEE; DE RUN, 2020). Dados do setor, obtidos nos relatórios do Sindicato das Mantenedoras do Ensino Superior (2016) e nas Sinopses Estatísticas (INEP, 2019), indicam crescimento de 7\% no número de IES entre 2015 e 2018 . Tal incremento veio acompanhado de crescimento ainda maior na oferta de cursos, que passaram de 33.501 em 2015 para 37.962 em 2018; 13\% a mais. Como resultado, em 2018 foram ofertadas 13.5 milhões de novas vagas de graduação no Brasil, das quais apenas 1/4 foram preenchidas (INEP, 2019).

Eventos mais recentes, como a crise econômica atual, advinda da pandemia gerada pela COVID-19, podem incrementar as pressões sobre as IES brasileiras, incluindo diminuição da entrada de novos alunos e aumento da inadimplência. Para Witze (2020), as debilidades estruturais de países em desenvolvimento, como baixa acessibilidade tecnológica e ambientes institucionais mais frágeis, devem aumentar tais pressões e fazer com que IES locais tenham que antecipar a implementação de seus planos estratégicos de longo-prazo para lidar com ambientes mais desafiadores pós-pandemia.

Consequentemente, os desafios atuais e futuros obrigam as IES privadas a buscar soluções de natureza mercadológica, como foco na qualidade de seus serviços, para melhorar seus resultados financeiros e operacionais (TEEROOVENGADUM et al., 2019). Atentar-se a construtos focados nas atitudes dos alunos sobre serviços prestados pode ajudar às IES na busca de vantagens competitivas e melhorias no desempenho, com potenciais impactos positivos na receita, já que a qualidade está associada com atributos que podem suprir 


\section{QUALIDADE PERCEBIDA POR ALUNOS DE INSTITUIÇÕES DE ENSINO SUPERIOR: EFEITOS DO TAMANHO \\ DA TURMA, GÊNERO E TEMPO DE EXPERIÊNCIA DO ALUNO \\ DOI: https://doi.org/10.5007/1983-4535.2021.e76356}

necessidades dos clientes, possibilitando obtenção de premium prices (ZEITHAML; PARASURAMAN; BERRY, 1990). Oliver (2015) sugere que, sobretudo em situações desafiadoras de negócios, as empresas deveriam focar na melhoria da satisfação e qualidade de seus serviços, o que impulsionaria as atitudes de recompra, recomendação e, consequentemente, os próprios resultados financeiros das empresas. A relação positiva entre os construtos qualidade percebida e atitudes do consumidor, como satisfação, lealdade, intenção de recomendação, e destes com o desempenho financeiro das empresas é reconhecida por diversos estudos em distintas áreas da indústria e de serviços (SORESCU; SORESCU, 2016; YE; DONG; LEE, 2017)

Estudos relacionados aos serviços educacionais também apontam relevância da gestão de qualidade para IES privadas (MANATOS; SARRICO; ROSA, 2017; PEIXOTO; AGUIAR, 2018) e necessidade da busca de melhorias nesta área para incrementar a qualidade percebida e demais construtos relevantes de consumo, como satisfação, intenção de recompra, recomendação, entre outros (PEDRO; MENDES; LOURENÇO, 2018; GUIMARÃES et al., TEEROOVENGADUM et al., 2019). Adicionalmente, requisitos operacionais e regulatórios do próprio setor obrigam, por meio de avaliações de órgãos oficiais, como INEP, a obtenção de índices mínimos de qualidade nos serviços. Tais avaliações podem afetar o desempenho financeiro das IES, tanto de forma indireta, com impactos sobre suas imagens, quanto de forma direta, com efeitos sobre a oferta de financiamentos públicos, por exemplo.

A maior compreensão da relação entre algumas variáveis e qualidade percebida dos alunos acerca dos serviços no ensino superior presencial privado pode gerar informações relevantes para tomada de decisões estratégicas e operacionais das IES e aumentar o valor dos achados desta pesquisa. Dentre as alternativas existentes, busca-se melhorar a compreensão geral sobre relações da qualidade percebida dos alunos com as seguintes variáveis: (i) tamanho da turma; (ii) gênero dos alunos e (iii) tempo de experiência no uso do serviço.

Além de servir como fonte de auxílio para tomada de decisões estratégicas das IES na alocações de recursos, a pesquisa pretende cobrir uma lacuna acadêmica percebida na área, com baixa presença de estudos em marketing, sobretudo, entre relações de qualidade percebida com tamanho da turma (WESTERLUND, 2008; BANDIERA; LARCINESE; RASUL, 2010). 


\section{REFERENCIAL TEÓRICO}

\subsection{QUALIDADE PERCEBIDA EM SERVIÇOS}

Fonte de debates desde o início do século XX, o tema qualidade e sua gestão está solidamente integrado à realidade de um número crescente de organizações neste novo século (MANATOS; SARRICO; ROSA, 2017). Prakash (2019) considera que o tema segue evoluindo, incluindo fases sobrepostas de conceptualização, expansão e revisões, enquanto Pedro, Mendes e Lourenço (2018) sugerem ainda existir desafios de mensuração e definição associados ao debate sobre qualidade percebida, que apresenta-se em estágio inicial em certas indústrias, como a de educação superior, por exemplo (TEEROOVENGADUM et al., 2019).

De qualquer forma, trabalhos iniciais relacionados ao conceito de qualidade em geral, já apontavam a necessidade de inclusão das percepções individuais dos consumidores no uso do item adquirido. Garvin (2002), por exemplo, menciona o caráter transcendente e a associação à percepção e satisfação do consumidor como algumas características do conceito de qualidade. Tais valores "humanísticos" percebidos em várias concepções, ou seja, aqueles oriundos de respostas subjetivas, relativistas e individuais, aproximam-se do conceito de qualidade percebida. Zeithaml (1988) desassocia o conceito de qualidade percebida daqueles mais próximos da objetividade, sugerindo que qualidade percebida seja um julgamento mais abstrato do consumidor sobre uma superioridade ou excelência geral de um produto ou serviço.

Parasuraman, Zeithaml e Berry (1988) reforçam a natureza de atitude e julgamento do consumidor. A confrontação das percepções dos consumidores e de suas expectativas durante consumo do serviço geraria o resultado da qualidade percebida, sendo fruto de avaliação, consciente e inconsciente, de percepções associadas às experiências prévias, alternativas, critérios ou expectativas dos consumidores (CASTLEBERRY; McINTYRE, 1992).

Cronin e Taylor (1992) sugerem, porém que qualidade percebida deva ser vista como uma atitude, passível de ser compreendida e mensurada por meio de modelo "adequaçãoimportância", ou seja, quando a atitude de cada indivíduo é definida pela avaliação da performance do serviço, de acordo com suas percepções de importância individuais.

A eficiente gestão da percepção de qualidade dos clientes, pode ser fonte relevante, de forma direta ou indireta, para obtenção de vantagens competitivas, melhorias nas demais atitudes de consumo e desempenho das empresas, sobretudo em ambientes de negócios dinâmicos e competitivos (CHEN; LIU, 2019). Desta forma, tampouco as IES privadas 


\section{QUALIDADE PERCEBIDA POR ALUNOS DE INSTITUIÇÕES DE ENSINO SUPERIOR: EFEITOS DO TAMANHO \\ DA TURMA, GÊNERO E TEMPO DE EXPERIÊNCIA DO ALUNO \\ DOI: https://doi.org/10.5007/1983-4535.2021.e76356}

deveriam privar-se de entender melhor as percepções dos alunos sobre a qualidade dos seus serviços (SUMAEDI et al., 2012; PEIXOTO; AGUIAR, 2018;).

\subsection{TAMANHO DA TURMA}

O conceito de tamanho da turma é, no ensino superior, dominado pelo debate de natureza pedagógica, principalmente no impacto didático em turmas com muitos alunos, centrando-se em itens objetivos da qualidade, como performance acadêmica dos alunos. Diversos obstáculos para o eficiente processo didático são apontados pelos autores com relação às turmas com muitos alunos, entre eles: maior dificuldade de interação entre docente e discente (VIEIRA-SANTOS; HENKLAIN, 2017), diminuições na motivação dos alunos, na aprendizagem efetiva e na sensibilidade docente aos problemas dos alunos (FOLEY; MANSIGLIA, 2014) e maior facilidade da utilização de metodologias ativas e menos tradicionais em turmas pequenas (WRIGHT; BERGOM; BARTHOLOMEW, 2019). Entretanto, não foram encontradas evidências que possibilitassem clara definição conceitual de tamanho das turmas ou tentativas de segmentação destas em quantidades de alunos.

Em pesquisas de marketing sobre comportamento do consumidor no ensino superior, o foco sobre o tema qualidade recaí, sobretudo, no impacto desta sobre a performance acadêmica dos alunos. Nestes trabalhos, conceitua-se tamanho da turma como quantidade de alunos matriculados e aptos para exame final em cada disciplina (MACHADO; VERAHERNANDEZ, 2008; BANDIERA; LARCINESE; RASUL, 2010; ANNEGUES; PORTO; FIGUEIREDO, 2020), ou como o número de alunos atendentes de cada disciplina (HUXLEY et al., 2018). Bandiera et al. (2010) entendem que o ensino superior tem, pela própria natureza mais autônoma de seu modelo didático, um volume de alunos por sala superior aos níveis de ensino iniciais e sua classificação entre turmas pequenas - até 19 alunos - ou grandes - acima de 104 alunos dá-se apenas pela distribuição da amostra pesquisada em seu estudo. Utilizando também critérios arbitrários e relacionados à amostra de sua pesquisa, Huxley et al. (2018) criam um grupo de "turmas que são pequenas" como sendo as turmas com até nove alunos atendentes, enquanto Westerlund (2008) utilizou apenas dois níveis diferentes, classificando como pequenas as turmas com 200 e grandes as turmas com 600 alunos. Por último, Sapelli e Illanes (2016), arbitrariamente, propuseram a divisão das diversas turmas de seu estudo em oito faixas com determinadas quantidades crescentes de alunos em cada uma delas. 


\section{QUALIDADE PERCEBIDA POR ALUNOS DE INSTITUIÇÕES DE ENSINO SUPERIOR: EFEITOS DO TAMANHO \\ DA TURMA, GÊNERO E TEMPO DE EXPERIÊNCIA DO ALUNO \\ DOI: https://doi.org/10.5007/1983-4535.2021.e76356}

No caso brasileiro, encontra-se também a utilização de medidas regulatórias para definições da variável tamanho da turma. Por exemplo, a Portaria CNE/CP $\mathrm{n}^{\circ} 10$, de 6/08/2009, estipula número máximo de 35 alunos por professor na Educação Superior, bem como observa-se existência de variedade de limites individuais do número máximo de alunos por classe em pareceres do Ministério da Educação (MEC) na autorização de abertura de cursos. Peixoto e Aguiar (2018), por exemplo, adotam limite de 50 alunos, com base em pareceres e liminares jurídicas, para dividir sua amostra de pesquisa entre turmas grandes e pequenas.

\subsection{GÊNERO}

O gênero é uma variável amplamente pesquisada em diversos campos de estudo, como, por exemplo, Psicologia e Marketing, incluindo longa tradição na investigação das diferenças de gênero em pesquisas sobre consumo, com potenciais contrastes não apenas nos objetos desejados, mas também no próprio processo decisório e utilização dos recursos compartidos de um casal (FERBER; LEE, 1974), bem como no processamento individual das informações relacionadas ao consumo (MEYERS-LEVY; MAHESWARAN, 1991). De maneira geral, entende-se que o gênero pode possuir potencial de impacto nas atitudes de compra. Tais diferenças podem ser encontradas em diversas etapas do processo, como, por exemplo: (i) na definição dos objetivos (MITCHEL; WALSH, 2004; KWUN, 2011); (ii) no processamento da mensagem (MEYERS-LEVY; MAHESWARAN, 1991); (iii) no processo de decisão (MITCHEL; WALSH, 2004); entre outros.

O conceito de qualidade percebida está associado, diretamente, com várias etapas do consumo, desde formação de expectativas que orientam a intenção de compra na pré-venda, até mesmo durante e após sua utilização, por meio das avaliações da performance dos serviços consumidos. Tal construto também possuí potencial de impacto em outras atitudes no póscompra, como recomendação e recompra (CRONIN; BRADY; HULT, 2000). Por conseguinte, entender fatores que impactam na qualidade percebida dos consumidores ganha relevância para atitudes gerencias das empresas sobre eficiência de suas vendas. Assim, trabalhos empíricos sobre o potencial de impacto do gênero na percepção de qualidade de distintos serviços vêm sendo realizados e, em muitos casos, atestam a significância do gênero do consumidor no resultado de tais percepções (SPATHIS; PETRIDOU; GLAVELI, 2004; MOKHLIS, 2012). 


\section{QUALIDADE PERCEBIDA POR ALUNOS DE INSTITUIÇÕES DE ENSINO SUPERIOR: EFEITOS DO TAMANHO \\ DA TURMA, GÊNERO E TEMPO DE EXPERIÊNCIA DO ALUNO \\ DOI: https://doi.org/10.5007/1983-4535.2021.e76356}

Apesar da importância do tema, não foi encontrada vasta produção acadêmica a respeito de eventuais diferenças significativas, advindas do gênero dos estudantes, na percepção de qualidade dos serviços das IES privadas, sobretudo no Brasil. Entre os estudos localizados, percebe-se tendência de validar a existência de diferenças nas percepções de qualidade dos estudantes de acordo com seus gêneros (SUMAEDI et al., 2012; ÇERA et al., 2018; KAUR; BHALLA, 2018), em linha com pressupostos conceituas acerca do tema.

\subsection{TEMPO DE EXPERIÊNCIA NO USO DE UM SERVIÇO OU PRODUTO}

O tempo de experiência no uso de um produto ou serviço também pode influenciar na satisfação e qualidade percebida do consumidor (BOLTON; DREW, 1991). Holbrook e Gardner (1993) sugerem que a duração do uso também afeta os componentes emocionais do consumo. No caso de sistemas de informação, por exemplo, o uso contínuo propicia alterações nas percepções e atitudes dos usuários, associado à relação positiva entre tempo de experiência de uso e lealdade dos consumidores (ZHANG et al., 2017). Consequentemente, tal fato aparenta possuir potencial de impacto sobre a própria manutenção do consumo do item ou serviço.

Baseado em estudos no campo da psicologia, como o modelo de ajustamento de opiniões de Hogart e Einhorn (1992), nota-se que o tempo de experiência no uso também pode afetar as percepções de qualidade dos consumidores devido à variabilidade existente nas múltiplas interações ao longo do tempo no consumo do serviço. O impacto das interações com experiências distintas inclú também fatores como ordem dos eventos - experiências negativas mais recentes tendem a ter maior impacto na percepção que experiências semelhantes mais antigas - e duração nas incorrências de tais variabilidades (GUSE et al., 2017).

Em serviços de uso prolongado e constantes, como ensino superior, a gestão e compreensão do processo de ajuste contínuo nas expectativas dos clientes, ou seja, a atualização frequente das expectativas geradas pelas interações com o provedor, é visto como fator mediador crítico entre satisfação e comprometimento com a lealdade dos alunos (PHAM; LAI, 2016). Alguns estudos empíricos sugerem potencial impacto do tempo de experiência nas percepções dos alunos como, por exemplo, a deterioração de percepções dos alunos do segundo ano sobre alguns aspectos relativos ao seu curso em uma IES pública britânica (WEBB; COTTON, 2019), ou percepções de qualidade mais negativas dos alunos 


\section{QUALIDADE PERCEBIDA POR ALUNOS DE INSTITUIÇÕES DE ENSINO SUPERIOR: EFEITOS DO TAMANHO \\ DA TURMA, GÊNERO E TEMPO DE EXPERIÊNCIA DO ALUNO \\ DOI: https://doi.org/10.5007/1983-4535.2021.e76356}

concluintes, quando comparadas com as percepções dos alunos ingressantes (POFFO; MARINHO, 2013).

\section{MODELO DE INVESTIGAÇÃO E FORMULAÇÃO DE HIPÓTESES}

A qualidade percebida dos serviços é uma atitude que pode ser capturada com avaliações da performance durante e após seu consumo (CRONIN; TAYLOR, 1992; BRADY; CRONIN; BRAND, 2002). Diversos são os fatores que podem influenciar nos resultados de qualidade percebida. De modo geral, este trabalho visa avaliar a significância da relação entre três variáveis distintas e qualidade percebida no serviço de educação superior.

Estudos acadêmicos no campo da Pedagogia apontam para uma relação negativa entre o tamanho da turma e a qualidade do ensino (FOLEY; MANSIGLIA, 2014; VIEIRASANTOS; HENKLAIN, 2017; WRIGHT; BERGOM; BARTHOLOMEW, 2019). Estudos independentes (Hanover Research Institute; 2010) também sugerem que turmas maiores prejudicam a qualidade de ensino ofertada e importantes rankings internacionais de qualidade de IES, como Times Higher Education [THE], The Guardian University Guide e QS World University Rankings, postulam que indicadores mais altos de "alunos por professor" sejam negativos para a qualidade ofertada pelas IES.

Por outro lado, há grande lacuna de estudos empíricos que comprovem essa relação (ANNEGUES; PORTO; FIGUEIREDO, 2020). O estudo realizou, para o período 2000-2020, buscas nos sites de pesquisa Web of Science - WoS, Google Scholar e na base SPELL da Associação Nacional de Pós-Graduação e Pesquisa em Administração - ANPAD utilizando, inicialmente, as seguintes palavras e suas variações: qualidade, qualidade percebida, ensino superior, universidade, quality, perceived quality, higher education, post-secondary education e university. $\mathrm{Na}$ sequência, incluíram-se os termos tamanho da turma, tamanho da sala, quantidade de alunos, class size e number of students. Entre os artigos encontrados, foram priorizados somente aqueles escritos em inglês, português ou espanhol e publicados em periódicos de maior impacto, categorizados com as notas Qualis-Periódicos B2, B1, A2 ou A1 para os artigos nacionais e com índices JCR ou H-Index para artigos internacionais.

No caso do Ensino Superior, nota-se que trabalhos avaliativos do impacto do tamanho da turma na qualidade do serviço, em sua grande maioria, relacionam tamanho da turma com a performance acadêmica dos alunos, e não com qualidade percebida dos serviços. Mesmo nesse caso, os resultados obtidos seguem inconclusivos (MIRANDA et al., 2015) sendo que 
fatores exógenos na alocação docente e discente podem ser algumas das causas das inconsistências destes achados (DE PAOLA; PONZO; SCOPPA, 2013; ANNEGUES; PORTO; FIGUEIREDO, 2020).

Alternativamente, porém em menor volume, alguns estudos, como Mandel e Süssmuth (2011) e Sapelli e Illanes (2016), buscaram analisar o impacto do tamanho da turma sobre certas percepções e atitudes dos alunos, sugerindo tendência favorável, por parte dos alunos, para classes menores. Entretanto, foram encontrados apenas poucos trabalhos focados na análise do tamanho da turma com qualidade percebida dos alunos. Westerlund (2008) avaliou a percepção dos alunos de uma IES sueca sobre algumas variáveis qualitativas e sugere que tamanho da classe tenha impacto negativo significativo sobre a qualidade percebida destes alunos. O construto qualidade percebida foi, porém, definido de maneira mais geral, sem utilização de escalas previamente testadas. Além deste trabalho, apenas outro estudo empírico, relacionando tamanho da turma e qualidade percebida dos alunos, foi encontrado na revisão bibliográfica. Peixoto e Aguiar (2018), realizaram pesquisa com 201 alunos de primeiro semestre em quatro disciplinas de uma IES privada brasileira e encontraram relação negativa entre qualidade percebida e tamanho da turma.

Apesar da pequena produção de evidências empíricas, a pesquisa sugere assumir o conceitual pedagógico para testar a seguinte hipótese: o aumento no tamanho da turma relaciona-se negativamente com a percepção de qualidade dos serviços nas IES privadas brasileiras (Hipótese 1).

O gênero do consumidor é uma variável amplamente pesquisada e possuí relevante potencial de impacto sobre a atitude de compra de serviços e produtos (MEYERS-LEVY; MAHESWARAN, 1991; MITCHEL; WALSH, 2004; KWUN, 2011), incluindo qualidade percebida. No caso do Ensino Superior, percebe-se que estudos sobre relação entre variáveis demográficas e percepções dos estudantes ainda não são o principal foco das pesquisas empíricas na área (SUMAEDI et al., 2012). Assim mesmo, nota-se certa tendência em apontar existência de diferenças, de acordo com o gênero dos estudantes, entre as percepções da qualidade percebida do serviço e satisfação (KAUR; BHALLA, 2018), a qualidade de forma geral (ÇERA et al., 2018), ou em apenas algumas de suas dimensões (SUMAEDI et al., 2012).

Com base no campo conceitual e nos estudos avaliados, o trabalho assume, como hipótese de pesquisa, que: o gênero dos estudantes está associado à ocorrência de diferenças 
significativas na qualidade percebida dos serviços nas IES privadas brasileiras (Hipótese 2).

O tempo de experiência na utilização de um serviço, sobretudo em serviços de natureza mais contínua, influencia na intensidade das desconfirmações e, por conseguinte, na qualidade percebida pelos consumidores (BOLTON; DREW; 1991; HOLBROOK; GARDNER, 1993; BOLTON, 1998). O serviço de educação superior presencial apresenta, por sua própria natureza, relação de continuidade entre IES e seus alunos. Webb e Cotton (2019) avaliaram o efeito da queda de performance acadêmica dos estudantes no segundo ano e observaram declínios significativos nas percepções dos alunos sobre a agradabilidade de seus cursos, além de incrementos nas frustrações de expectativas e nas intenções de evasão. Por conseguinte, o trabalho irá assumir a hipótese conceitual de que: o tempo de experiência na utilização do serviço educacional superior gera diferenças significativas na qualidade percebida dos serviços nas IES privadas brasileiras (Hipótese 3).

\section{METODOLOGIA}

A natureza da pesquisa empreendida é quantitativa e referente a uma população formada por alunos de cursos presenciais devidamente matriculados no primeiro semestre letivo do ano de 2017 em três IES privadas brasileiras. As IES estão localizadas em três distintos Estados do Nordeste brasileiro e possuem grande relevância, em termos de total de alunos, em suas cidades. Todas ofertam ampla gama de cursos de graduação nos níveis de Bacharelado, Licenciatura e Tecnólogo, tanto presencial quanto online. Suas identidades serão preservadas, utilizando-se a nomenclatura IES 1, IES 2 e IES 3 para designá-las nesse estudo.

O processo de seleção da amostra foi não probabilístico, realizado por julgamento e conveniência, procurando refletir proporcionalmente o universo total da população das IES, tanto em distribuição dos cursos, quanto de séries existentes. Como cada aluno poderia estar matriculado em mais que uma turma, buscou-se também, com análises de seleção das turmas da amostra, privilegiar respostas únicas por aluno. Desta forma, foram coletados dados em 61 turmas, de tamanhos variados, incluindo a menor com oito alunos e a maior com 114 membros, sendo 2.934 o total de alunos das 61 turmas.

A mensuração da variável qualidade percebida centrou-se na escala SERVPREF (CRONIN; TAYLOR, 1992), amplamente utilizada para avaliações deste construto em trabalhos de Marketing. Assim como no trabalho de Cronin e Taylor (1992) a pesquisa 


\section{QUALIDADE PERCEBIDA POR ALUNOS DE INSTITUIÇÕES DE ENSINO SUPERIOR: EFEITOS DO TAMANHO \\ DA TURMA, GÊNERO E TEMPO DE EXPERIÊNCIA DO ALUNO \\ DOI: https://doi.org/10.5007/1983-4535.2021.e76356}

utilizou, como ponto inicial, a escala SERVQUAL revista (PARASURAMAN; ZEITHAML; BERRY, 1991), com 22 variáveis divididas em cinco dimensões, descritas na Tabela 1. Apesar de propor a unidimensionalidade da escala, Cronin e Taylor (1992) sugerem possibilidade de diferentes comportamentos de fatoração, dependendo do setor de serviços. Estudos preliminares no segmento de Ensino Superior mostram significativo potencial de confiabilidade das dimensões originais da escala SERVQUAL (PEIXOTO; AGUIAR, 2018). Por conseguinte, decidiu-se manter as cinco dimensões nas análises do trabalho. Preliminarmente, também se procedeu com a tradução para o português da escala. Para evitar discrepâncias de entendimento, a escala foi retraduzida para o inglês e verificada contra a escala original.

Tabela 1 Dimensões da SERVQUAL

\begin{tabular}{l|l}
\hline Dimensão & Abrangência / Definição \\
\hline Tangibilidade & Instalações, equipamento e aparência do pessoal \\
\hline Confiabilidade & Habilidade em prestar o serviço prometido de forma acurada e confiável \\
\hline Responsividade & Desejo de ajudar os clientes e fornecer um serviço com prontidão \\
\hline Garantia/Segurança & Conhecimento e cortesia dos empregados e sua habilidade de inspirar confiança \\
\hline Empatia & Cuidado, atenção individualizada da empresa para seus clientes \\
\hline
\end{tabular}

Fonte: adaptado de Peixoto \& Aguiar (2018).

Com objetivo de aumentar a aderência e previsibilidade da SERVPERF à proposta desta pesquisa, buscou-se adaptação da escala original ao contexto estudado (PARASURAMAN; ZEITHAML; BERRY, 1988); ou seja, uma adequação desta à realidade do ensino educacional superior brasileiro e, posteriormente, até mesmo ao ambiente específico das três IES no qual ela foi aplicada. Como primeiro passo, realizou-se revisão bibliográfica da utilização da SERVQUAL e da SERVPERF em estudos no ensino superior nacional e, em conjunto com os especialistas acadêmicos das três IES, uma avaliação do instrumento para inclusão de termos e itens relevantes para o contexto da pesquisa. A primeira versão foi então submetida para pré-teste com 90 alunos de três turmas distintas de uma das IES da pesquisa no segundo semestre de 2016. Os resultados obtidos foram testados para confirmação de dimensionalidade, confiabilidade e validade das variáveis e serviram como fonte para definição da versão utilizada no presente estudo. Como consequência: (i) o instrumento manteve as cinco dimensões originais; (ii) uma variável da dimensão "Confiabilidade" foi excluída, por não encontrar aderência ao serviço de ensino; (iii) três variáveis da dimensão "Empatia" foram consolidadas em uma, facilitando compreensão do público da amostra e; (iv) adicionou-se uma variável à dimensão "Empatia" sobre atividades 


\section{QUALIDADE PERCEBIDA POR ALUNOS DE INSTITUIÇÕES DE ENSINO SUPERIOR: EFEITOS DO TAMANHO \\ DA TURMA, GÊNERO E TEMPO DE EXPERIÊNCIA DO ALUNO \\ DOI: https://doi.org/10.5007/1983-4535.2021.e76356}

extraclasse, vista como importante pelos especialistas da área. Entende-se assim que, a integridade da escala original foi mantida.

Como resultado das análises e verificação da escala, foi gerado um questionário com 20 variáveis representando as cinco dimensões da qualidade percebida, além de seis perguntas de caráter sociodemográfico e estudantil, vinculadas à escala Likert de cinco pontos.

O estudo utilizou método de comunicação estruturado não-disfarçado, com distribuição de questionários para respondentes voluntários e conscientes dos objetivos da pesquisa, sem oferta de benefícios e penalidades à população amostral. Este trabalho garantiu total anonimato dos respondentes, fato fundamental para evitar atitudes prejudiciais à pesquisa, como constrangimentos e medos de represálias por parte dos respondentes, sobretudo tratando-se de serviço contínuo e fundamental para sociedade e para muitos dos próprios alunos das IES.

Objetivando diminuir riscos de vieses comportamentais e, ao mesmo tempo, aumentar a solidez dos dados, a pesquisa elegeu, como momento crítico para aplicação dos surveys, o final do semestre, uma semana antes das provas finais, momento já com sólida relação entre "o consumidor e o serviço consumido" e, ao mesmo tempo, sem risco de contágio pelos eventuais resultados de aprovação acadêmica ou não dos alunos pesquisados. O questionário foi distribuído e recolhido por funcionários das próprias IES, sem intervenção do professor da disciplina avaliada. A orientação para preenchimento e objetivo da pesquisa foi comunicada oralmente pelos funcionários e por escrito na parte inicial do questionário. As variáveis Gênero e Tempo de Experiência foram coletadas nesse mesmo questionário, sendo que, para esta última, adotou-se a premissa de estudos acadêmicos e dos especialistas das IES pesquisadas para definir "calouros" como alunos no primeiro ou segundo semestre de seus cursos e "Veteranos" como alunos matriculados a partir do terceiro semestre do seu curso.

Em linha com estudos empíricos, considerou-se número de alunos matriculados como tamanho da turma. Tais dados foram obtidos com cada IES participante. De acordo com o trabalho de Peixoto e Aguiar (2018), esta pesquisa adota o critério de 50 alunos como limite para turmas consideradas pequenas, sendo turmas com mais de 50 alunos consideradas grandes. 


\section{ANÁLISE E DISCUSSÃO DOS RESULTADOS}

Os processos metodológicos da pesquisa visaram garantir maior confiabilidade possível do instrumento de coleta. Assim mesmo, foram realizados testes adicionais para garantir a dimensionalidade e confiabilidade do modelo implementado. Inicialmente, procedeu-se com a limpeza da base, sendo retirados questionários recebidos de forma incompleta ou com respostas inadequadas, além da exclusão de cinco turmas devido à observância de inconsistências em seus processos de coleta. Ao final, validaram-se $90,5 \%$ de todos os questionários recebidos.

Com base nos dados válidos, foram realizados testes de análise fatorial exploratória, que confirmaram a estrutura dos fatores proposta, com as 20 variáveis divididas nas cinco dimensões originais devidamente validadas no pré-teste alcançando a medida Kayser-MeyerOlkin (KMO) igual a 0,969 com significância para $p<0,001$ no teste de esfericidade de Bartlett (aprox. Qui-quadrado $=19398,973$ e df $=190$ ). As cinco dimensões também tiveram suas confiabilidades testadas e validadas pelo alfa de Cronbach, superior ao limite de 0,7 comumente aceito em pesquisas similares, conforme demonstrado na Tabela 2.

Tabela 2 Resultados do Alfa de Cronbach para cada dimensão do construto qualidade percebida

\begin{tabular}{l|c|c|c|c|c}
\hline \multicolumn{1}{c|}{ Dimensão } & Tangibilidade & Confiab. & Respons. & Garantia & Empatia \\
\hline $\begin{array}{l}\text { Alfa de } \\
\text { Cronbach }\end{array}$ & 0,800 & 0,825 & 0,893 & 0,856 & 0,824 \\
\hline
\end{tabular}

Fonte: elaboração própria.

Após exclusão de respostas não adequadas, foi obtida uma amostra de 1.379 questionários válidos, oriundos de 56 turmas distintas, distribuídas por 51 professores, obtendo-se taxas de respostas válidas superiores a 35\% em todas as IES, conforme Tabela 3.

Tabela 3 Distribuição dos alunos, turmas e professores por IES

\begin{tabular}{c|c|c|c|c|c}
\hline Dimensão & Qtde Professores & Qtde Turmas & $\begin{array}{c}\text { Tamanho } \\
\text { População }\end{array}$ & $\begin{array}{c}\text { Respostas } \\
\text { Válidas }\end{array}$ & $\begin{array}{c}\text { \% Respostas } \\
\text { Válidas }\end{array}$ \\
\hline IES1 & 14 & 14 & 841 & 303 & $36,0 \%$ \\
\hline IES2 & 18 & 21 & 907 & 380 & $41,9 \%$ \\
\hline IES3 & 19 & 21 & 1.146 & 696 & $60,7 \%$ \\
\hline TOTAL & 51 & 56 & 2.894 & 1.379 & $47,7 \%$ \\
\hline
\end{tabular}

Fonte: elaboração própria.

Com relação à faixa etária, 37,1\% dos alunos respondentes tem até 20 anos, 46,6\% entre 21 e 30 anos, 14,7\% acima de 30 anos e 1,5\% não responderam. Destaca-se também o 
número de alunos que alegaram ter financiamento (FIES) ou PROUNI: 29,8\% e 11,9\% respectivamente. Nas faixas de renda familiar, 39,9\% relatam ter renda até R\$ 1.765,00, enquanto 35,6\% encontram-se na faixa entre $\mathrm{R} \$ 1.765,01$ até $\mathrm{R} \$ 4.076,00$. A faixa acima de $\mathrm{R} \$ 4.076,00$ engloba $18,2 \%$ dos respondentes, com os restantes $6,3 \%$ preferindo não responder à questão. Cabe ressaltar que o perfil socioeconômico e demográfico alcançado na amostra está em linha com o perfil da população total das três IES da pesquisa, segundo seus próprios gestores.

Associado às variáveis necessárias para o trabalho, a divisão dos respondentes entre os grupos de interesse para as hipóteses de pesquisa é apresentada na Tabela 4, assim como suas médias e desvios-padrões.

Tabela 4 Número de alunos por grupo

\begin{tabular}{c|l|c|c|c}
\hline Variável & \multicolumn{1}{|c|}{ Grupo } & N & Média & Desvio-padrão \\
\hline $\begin{array}{c}\text { Tamanho da Turma } \\
\text { (H1) }\end{array}$ & Turma com até 50 alunos & 505 & 4,427 & 0,710 \\
& Turma com mais de 50 alunos & 874 & 4,402 & 0,549 \\
\hline $\begin{array}{c}\text { Gênero } \\
(\mathbf{H 2})\end{array}$ & Masculino & 664 & 4,385 & 0,643 \\
\hline $\begin{array}{c}\text { Tempo de Experiência } \\
(\mathbf{H 3})\end{array}$ & Feminino & 715 & 4,436 & 0,582 \\
\hline
\end{tabular}

Fonte: elaboração própria.

A preparação de cada grupo de análise seguiu suas considerações teóricas, respeitando também as características da população-alvo da pesquisa. Sendo assim, alunos de turmas dos primeiros e segundo semestre foram qualificados como "calouros" ou ingressantes, enquanto os representantes das demais turmas foram qualificados como "veteranos". Com relação ao gênero, ofereceu-se, a possibilidade fechada de duas opções: masculino ou feminino. Não respondentes foram respeitados, sendo suas respostas excluídas desta pesquisa, em particular. A variável Tamanho da Turma respeitou o critério de 50 alunos como limite para definição da divisão entre turmas, gerando 33 turmas com até 50 alunos e 23 com mais de 50 alunos.

Parasuraman, Zeithaml e Berry (1988) consideram a SERVQUAL como uma escala que pode servir como instrumento de gestão para avaliar a qualidade percebida dos serviços prestados por meio de cada dimensão, devendo-se, para isso, utilizar a média entre as variáveis dessa dimensão. Adicionalmente, sugerem que a média entre as cinco dimensões serviria como avaliação da qualidade percebida geral do serviço. Sendo assim, esta pesquisa assume a média simples entre variáveis do questionário como proxy da Percepção de 


\section{QUALIDADE PERCEBIDA POR ALUNOS DE INSTITUIÇÕES DE ENSINO SUPERIOR: EFEITOS DO TAMANHO \\ DA TURMA, GÊNERO E TEMPO DE EXPERIÊNCIA DO ALUNO \\ DOI: https://doi.org/10.5007/1983-4535.2021.e76356}

Qualidade para todos seus testes realizados, tanto para percepção geral quanto para percepções de cada dimensão.

Visto que a distribuição da variável qualidade percebida, utilizada como variável dependente, não era normal (apresentando $p$-valor $<0,001$ para os testes de normalidade de Kolmogorov-Smirnov, Shapiro-Wilk e Anderson-Darling) e a variância dela entre os diversos grupos analisados não era homogênea, decidiu-se pela utilização de testes não paramétricos (Mann-Whitney U) para explorar diferenças na qualidade percebida entre os grupos definidos pelas hipóteses de pesquisa. Os resultados encontrados para as diferenças de média entre os grupos são apresentados na Tabela 5.

Tabela 5 Resultados do teste de Mann-Whitney U para cada hipótese de pesquisa

\begin{tabular}{l|c}
\hline \multicolumn{1}{c|}{ Hipótese } & $p$-valor \\
\hline $\begin{array}{l}\text { H1: o aumento no tamanho da turma relaciona-se negativamente com a percepção de qualidade dos } \\
\text { serviços nas IES privadas brasileiras. }\end{array}$ & 0,001 \\
\hline $\begin{array}{l}\text { H2: o gênero dos estudantes está associado à ocorrência de diferenças significativas na qualidade } \\
\text { percebida dos serviços nas IES privadas brasileiras. }\end{array}$ & 0,211 \\
\hline $\begin{array}{l}\text { H3: o tempo de experiência na utilização do serviço educacional superior gera diferenças significativas } \\
\text { na qualidade percebida dos serviços nas IES privadas brasileiras. }\end{array}$ & 0,025 \\
\hline
\end{tabular}

Fonte: elaboração própria.

Conforme os $p$-valores dispostos na Tabela 5 e considerando nível de significância de 0,05 , foram encontradas diferenças significativas entre percepções de qualidade de alunos em turmas menores e maiores (H1) e entre alunos com diferentes tempos de experiência no ensino superior (H3). Com respeito ao gênero (H2), os dados utilizados não fornecem evidências suficientes para indicar diferenças na percepção de qualidade, de forma geral, entre alunos de gênero masculino e feminino.

Mesmo com a média geral das 20 variáveis representando a qualidade percebida dos serviços das IES, esta pesquisa também objetiva fornecer informação adicional e útil para gestão das IES. Sendo assim, a análise individual das dimensões pode oferecer subsídios para melhor entendimento das fontes potenciais das diferenças de percepções gerais encontradas pela pesquisa e endereçar ações mais eficientes das IES em suas alocações de recursos.

Os $p$-valores da Tabela 6 mostram, com nível de significância de 0,05 , que a dimensão de Tangibilidade possuí diferenças significativas entre qualidade percebida e tamanho da turma e tempo de experiência, sem resultado significativo para o gênero. A dimensão de Confiabilidade apresenta significância apenas para tempo de experiência, enquanto as dimensões de Responsividade e Empatia sugerem existência de diferenças significativas da percepção de qualidade apenas com relação ao tamanho da turma. Por último, os $p$-valores da 


\section{QUALIDADE PERCEBIDA POR ALUNOS DE INSTITUICOÕES DE ENSINO SUPERIOR: EFEITOS DO TAMANHO \\ DA TURMA, GÊNERO E TEMPO DE EXPERIÊNCIA DO ALUNO \\ DOI: https://doi.org/10.5007/1983-4535.2021.e76356}

dimensão Garantia, apresentam diferenças significativas da qualidade percebida tanto para tamanho da turma quanto para gênero, sem significância para com tempo de experiência.

Tabela 6 Resultados do teste Mann-Whitney U para cada hipótese da pesquisa por dimensão

\begin{tabular}{l|c|c|c|c|c|c}
\hline \multicolumn{1}{c|}{ Variável } & $\begin{array}{c}\boldsymbol{p} \text {-valor } \\
\text { Geral }\end{array}$ & $\begin{array}{c}\boldsymbol{p} \text {-valor } \\
\text { Tangib. }\end{array}$ & $\begin{array}{c}\boldsymbol{p} \text {-valor } \\
\text { Confiab. }\end{array}$ & $\begin{array}{c}\boldsymbol{p} \text {-valor } \\
\text { Respons }\end{array}$ & $\begin{array}{c}\boldsymbol{p} \text {-valor } \\
\text { Garantia }\end{array}$ & $\begin{array}{c}\boldsymbol{p} \text {-valor } \\
\text { Empatia }\end{array}$ \\
\hline Tamanho da turma (H1) & 0,001 & 0,020 & 0,063 & 0,001 & 0,000 & 0,035 \\
\hline Gênero(H2) & 0,211 & 0,475 & 0,974 & 0,358 & 0,027 & 0,139 \\
\hline Tempo de Experiência (H3) & 0025 & 0,002 & 0,003 & 0,059 & 0,248 & 0,156 \\
\hline
\end{tabular}

Fonte: elaboração própria.

\subsection{DISCUSSÃO DOS RESULTADOS}

O p-valor de 0,001 (Tabela 5) do Teste de Mann-Whitney U sugere a confirmação da Hipótese 1 de que o aumento no tamanho da turma relaciona-se negativamente com a percepção de qualidade dos serviços nas IES privadas brasileiras. Entre as 56 turmas das três IES testadas, percebe-se que as diferenças entre percepções de qualidade dos alunos de turmas com mais de 50 alunos são, significativamente, menores que nas turmas até 50 alunos.

Apesar de diversos artigos acadêmicos tratarem dos problemas e desafios da educação em turmas grandes, a relação entre tamanho da turma e qualidade percebida encontra pouca referência em trabalhos de pesquisa. Peixoto e Aguiar (2018) avaliaram uma amostra de quatro turmas e sugerem ocorrência de relação negativa entre essas variáveis. As análises desta pesquisa apresentam resultados em linha com tais achados. Adicionalmente, percebe-se significância da relação negativa entre estas variáveis também em quatro das cinco dimensões de qualidade avaliadas. Apenas a dimensão de Confiabilidade, com p-valor de 0,063 apresenta alguma possibilidade de semelhanças de percepções de qualidade entre os alunos de distintos tamanhos de turma. As quatro dimensões restantes apresentam diferenças de percepções significativas e, em conjunto com o resultado da percepção geral, sugerem que as IES devam prestar atenção generalizada em quase todos aspectos de seus serviços para alunos de turmas com mais de 50 estudantes matriculados, sobretudo ao perceber-se o risco associado entre baixa percepção de qualidade e a eventual lealdade dos alunos (ZEITHAML; PARASURAMAN; BERRY, 1990), que poderia ser identificado com o desafio de retenção destes.

Os resultados obtidos também validam a Hipótese 3 da pesquisa, ou seja, o tempo de experiência na utilização do serviço educacional superior gera diferenças significativas na 


\section{QUALIDADE PERCEBIDA POR ALUNOS DE INSTITUIÇÕES DE ENSINO SUPERIOR: EFEITOS DO TAMANHO \\ DA TURMA, GÊNERO E TEMPO DE EXPERIÊNCIA DO ALUNO \\ DOI: https://doi.org/10.5007/1983-4535.2021.e76356}

qualidade percebida dos serviços nas IES privadas brasileiras. O declínio das percepções de qualidade com tempo de experiência dos alunos em cursos do ensino superior já havia sido sugerido por algumas pesquisas, verificando gradual processo de desmotivação dos alunos (POFFO; MARINHO, 2013), incluindo risco de impactos negativos sobre o comportamento futuro e potencial de recompra para as IES (TOMASSINI; AQUINO; CARVALHO, 2008).

Vale ressaltar, em análise mais detalhada por dimensão, que esse declínio nas percepções de qualidade dos "veteranos" possa estar mais associado com fatores e dimensões específicas. Apesar das médias das percepções dos "calouros" serem superiores às dos "veteranos" em todas as dimensões, os resultados sugerem significância nas diferenças apenas nas dimensões de Tangibilidade e Confiabilidade. A verificação mais detalhada destes resultados pode auxiliar as IES na implementação de ações mitigadoras mais eficientes sobre o declínio da qualidade percebida pelo tempo de experiência dos alunos.

Finalmente, resultados obtidos não comprovaram a Hipótese 2, sugerindo que o gênero dos estudantes não esteja associado à ocorrência de diferenças significativas na qualidade percebida dos serviços. Porém, assim como no caso da variável tempo de experiência, análises mais detalhadas por cada dimensão da qualidade percebida devem ser empregadas. Sumaedi et al. (2012) e Joseph, Yakhou e Stone (2005) já apontavam para eventuais diferenças entre gêneros dos estudantes apenas para certas dimensões ou itens da qualidade percebida. De maneira similar, foi encontrada diferença significativa em apenas uma determinada dimensão do construto (Garantia/Segurança), sugerindo que a diferença de gênero dos estudantes possa gerar resultados significativos sobre itens ou dimensões específicas da qualidade percebida.

\section{CONSIDERAÇÕES FINAIS}

As principais contribuições teóricas deste trabalho se encontram, sobretudo, nas análises empíricas e achados para itens relevantes na sociedade, como o Ensino Superior, e para áreas de pesquisa em Marketing, como qualidade percebida e suas relações com tamanho da turma, gênero e tempo de experiência de uso dos consumidores. A tendência de aumento do tamanho das turmas no Ensino Superior privado presencial brasileiro acarreta riscos à qualidade dos serviços nas IES e, consequentemente, também à sua performance financeira (ANNEGUES; PORTO; FIGUEIREDO, 2020). Assim mesmo, dados e pesquisas sobre essa tendência e seus impactos sobre serviços prestados pelas IES ainda são escassos (HUXLEY et 


\section{QUALIDADE PERCEBIDA POR ALUNOS DE INSTITUIÇÕES DE ENSINO SUPERIOR: EFEITOS DO TAMANHO \\ DA TURMA, GÊNERO E TEMPO DE EXPERIÊNCIA DO ALUNO \\ DOI: https://doi.org/10.5007/1983-4535.2021.e76356}

al., 2018). Para o segmento de Ensino Superior, há certa carência de estudos também sobre as relações entre o tempo de experiência de uso e gênero dos alunos com a qualidade percebida dos serviços.

A cobertura de tais lacunas acadêmicas, junto com os demais achados da pesquisa, também possuem impacto prático, ao possibilitar melhor orientação da estratégia competitiva e alocação de recursos das IES, objetivando diminuir riscos e incrementar oportunidades de efetiva gestão da qualidade de seus serviços (MANATOS; SARRICO; ROSA, 2017; KAUR; BHALLA, 2018). A melhor compreensão dos resultados encontrados nesta pesquisa, que demonstram relações significativas entre qualidade percebida pelos alunos e tamanho da turma, tempo de experiência e gênero (na dimensão Garantia/Segurança) deve servir como alerta e orientar as IES privadas nacionais na busca por melhorias na prestação de seus serviços, visto o potencial do impacto da qualidade percebida sobre as demais atitudes de seus alunos e, por fim, sobre seus próprios resultados financeiros (JOSEPH; YAKHOU; STONE, 2005; PEDRO; MENDES; LOURENÇO, 2018; GUIMARÃES et al., 2019).

No campo teórico, a pesquisa possibilita maior desenvolvimento do debate sobre os resultados encontrados e poucos explorados da área, que sugerem relação negativa do tamanho da turma e da experiência do tempo de uso com qualidade percebida. Também traz necessidade de aprofundamento da compreensão dos resultados não significativos para variável gênero dos estudantes, entendendo a demanda por maior detalhamento e avaliação à nível das dimensões formativas do construto qualidade percebida no ensino superior presencial privado.

No caso das IES, apesar da impossibilidade de generalizações devido ao seu escopo, a pesquisa permite questionamento estratégico e busca de soluções para o potencial declínio da percepção de qualidade de seus alunos à medida que progridem em seus estudos. Entender o processo de ajuste contínuo das expectativas dos alunos e criar laços mais emotivos e duradouros com eles deve ser fator crítico para serviços de longa duração, relevantes e constantes, como o caso da educação superior (PHAM; LAI, 2016).

As IES devem buscar também entender e gerenciar seus desafios operacionais e estratégicos, como a tendência do aumento de alunos por classe. Os resultados deste estudo demonstram existência de menores percepções de qualidade quando os serviços são ofertados em salas superiores a 50 alunos, gerando assim um risco sobre o desempenho atual e futuro das instituições deste setor. 
Entende-se que, além das limitações de generalização, o estudo tampouco excluiu a possibilidade do impacto de fatores endógenos na formação das turmas da amostra; ou seja, a eventual alocação não aleatória de alunos, professores e outros recursos pode ter influenciado os resultados obtidos. Também cabe notar a possibilidade de inclusões de indivíduos em grupos distintos aos seus, sobretudo no caso da separação entre "veteranos" e "calouros" que possam estar em semestres distintos aos de seus semestres utilizados na pesquisa.

Por outro lado, percebe-se, vasto campo de possibilidades para futuras pesquisas, sobretudo pela carência de maior volume de trabalhos na área. A adição de outros construtos relevantes para o consumo e performance das IES, como satisfação, recompra e recomendação, por exemplo, e suas relações com tamanho da turma podem adicionar valor ao debate e à gestão das instituições do setor. Finalmente, igualmente relevante seria melhorar a compreensão da causalidade na percepção de qualidade dos alunos, buscando-se entender quais variáveis e em qual medida podem impactar a qualidade percebida dos serviços.

\section{REFERÊNCIAS}

ANNEGUES, C.A.: PORTO, S.; FIGUEIREDO, E. Tamanho da Turma e Desempenho Acadêmico dos Universitários: evidência para a UFPB. Estudos Econômicos, n.50, p. 99$124,2020$.

BANDIERA, O.; LARCINESE, V.; RASUL, I. Heterogeneous Class Size Effects. The Economic Journal, n.120, p.1365-1398, 2010.

BOLTON, R.N. A Dynamic Model of Duration of the Customer's Relationship with Continuous Service Provider: Role of Satisfaction. Marketing Science, n.17, p.45-65, 1998.

BOLTON, R.N., DREW, J.H. A Longitudinal Analysis of the Impact of Service Changes on Customer Attitudes. Journal of Marketing, n. 55, v.1, p. 1-9, 1991.

BRADY, M.K., CRONIN, J.J., BRAND, R.R. Performance-only measurement of service quality: a replication and extension. Journal of Business Research, v. 55, p. 17-31, 2002.

CASTLEBERRY, S., \& McINTYRE, F.S. Consumers' Quality Evaluation Process. Journal of Applied Business Research, 8(3), 74-82, 1992.

ÇERA, G.; CEPEL, M.; ZAKUTNA, S.; ROSZA, Z. Gender differences in the perception of the university education quality as applied to entrepreneurial intention. Journal of

International Studies, n. 11, v. 3, p. 147-160, 2018.

CHEN, C-M., LIU, H-M. The moderating effect of competitive status on the relationship between customer satisfaction and retention. Total Quality Management \& Business Excellence, v. 30, p. 721-744, 2019. 
CRONIN, J.J.; BRADY, M.K.; HULT, G.T.M. Assessing the Effects of Quality, Value, and Customer Satisfaction on Consumer Behavioral Intentions in Service Environments. Journal of Retailing, v. 76, n. 2, p. 193-218, 2000.

CRONIN, J.J.; TAYLOR, S.A. Measuring Service Quality: A Reexamination and Extension. Journal of Marketing, v. 56, n. 3, p. 55-68, 1992.

DE PAOLA, M.; PONZO, M.; SCOPPA, V. Class size effects on student achievement: heterogeneity across abilities and fields. Education Economics, v. 21, p. 135-153, 2013.

FERBER, R.; LEE, L.C. Husband-wife influence in family purchasing behavior. Journal of Consumer Research, v. 1, n.1, p. 43-50, 1974.

FOLEY, A.R.; MASINGILA, J.O. Building capacity: challenges and opportunities in large class pedagogy (LCP) in Sub-Saharan Africa. Higher Education, v. 67, p. 797-808, 2014.

GARVIN, D. A. Gerenciando a Qualidade: a visão estratégica e competitiva. Rio de Janeiro, Qualitymark, 2002

GUIMARÃES, J.C.F.; SEVERO, E.A.; NOBREGA, K.C.; LEONE, M.N.C.P.G. A influência da inovação no ensino, qualidade e comprometimento sobre a retenção de alunos no ensino superior. Revista Gestão Universitária na América Latina, v. 12, n. 1, p. 249-269, 2019.

GUSE, D.; WEISS, B.; HAASE, F.; WUNDERLICH, A.; MÖLLER, S. Multi-episodic perceived quality for one session of consecutive usage episodes with a speech telephony service. Quality User Experience, v. 2, n. 8, p. 1-16, 2017.

HANOVER RESEARCH INSTITUTE. Strategies for Teaching Large Undergraduate Classes. Washington, 2010.

HOGART, R.M.; EINHORN, H.J. Order effects in belief updating: the Belief-Adjustment model. Cognitive Psychology, v. 24, p. 1-55, 1992.

HOLBROOK, M.B., GARDNER, M.P. An Approach to Investigating the Emotional Determinants of Consumption Durations: Why Do People Consume What They Consume for as Long as They Consume It? Journal of Consumer Psychology, v. 2, p. 123-142, 1993.

HOPER - EDUCAÇÃO. Análise Setorial da Educação Superior Privada (11. ed.), 2018.

HUXLEY, G.; MAYO, J.; PEACEY, M.W.; RICHARDSON, M. Class Size at University. Fiscal Studies, v. 39, n. 2, p. 241-264, 2018.

INEP. Sinopse Estatística da Educação Superior 2011 a 2018. [online], 2012 a 2019. [citado 02/10/2019]. Disponível em: http://portal.inep.gov.br/basica-censo-escolar-sinopsesinopse. 
JOSEPH, M.; YAKHOU, M.; STONE, G. An education institution's quest for service quality: custormers' perspective. Quality Assurance in Education, v. 13, n. 1, p. 66-82, 2005.

KAUR, H.; BHALLA, G.S. Determinants of effectiveness in public higher educationstudents' viewpoint. International Journal of Educational Management, v.32, p. 11351155, 2018.

KWUN, D.J. Effects of campus foodservice attributes on perceived value, satisfaction, and consumer attitude: A gender-difference approach. International Journal of Hospitality Management, v. 30, p. 252-261, 2011.

LIM, W.M.; JEE, T.W.; DE RUN, E.C. Strategic brand management for higher education institutions with graduate degree programs: empirical insights from the higher education marketing mix. Journal of Strategic Marketing, v. 28, n. 3, p. 225-245, 2020.

MACHADO, M.P.; VERA-HERNANDEZ, M. Does Class Size Affect the Academic Performance of First Year College Students? Mimeo University College London, 2008.

MANATOS, M.J.; SARRICO, C.S.; ROSA, M.J. The integration of quality management in higher education institutions: a systematic literature review. Total Quality Management \& Business Excellence, v. 28, n. 1-2, p. 159-175, 2017.

MANDEL, P.; SÜSSMUTH, B. Size matters. The relevance and Hicksian surplus of preferred class size. Economics of Education Review, v. 30, p. 1073-1084, 2011.

MEYERS-LEVY, J.; MAHESWARAN, D. Exploring differences in Males' and Females' processing strategies, Journal of Consumer Research, v. 18, p. 63-70, 1991.

MIRANDA, G.J.; LEMOS, K.C.S.; OLIVEIRA, A.S.; FERREIRA, M.A. Determinantes do Desempenho Acadêmico na Área de Negócios. Meta:Avaliação, v. 7, p. 175-209, 2015.

MITCHELL, V.; WALSH, G. Gender differences in German consumer decision-making styles. Journal of Consumer Behaviour, v. 3, p. 331-346, 2004.

MOKHLIS, S. The Influence of Service Quality on Satisfaction: A Gender Comparison. Public Administration Research, v.1, n. 1, p. 103-112, 2012.

OLIVER, R.L. Satisfaction: a behavioral perspective on the consumer (2. Ed). New York, Routledge, 2015.

PARASURAMAN, A.; ZEITHAML, V.A.; BERRY, L.L. SERVQUAL: A Multiple-Item Scale for Measuring Consumer Perceptions of Service Quality. Journal of Retailing, v. 64, n. 1, p. 12-40, 1988.

PARASURAMAN, A.; ZEITHAML, V.A.; BERRY, L.L. Refinement and Reassessment of the SERVQUAL Scale. Journal of Retailing, v. 67, n. 4, p. 420-450, 1991. 
PEDRO, E.; MENDES, L.; LOURENÇO, L. Perceived service quality and students' satisfaction in higher education: the influence of teaching methods. International Journal for Quality Research, v. 12, n. 1, p. 165-192, 2018.

PEIXOTO, M.R.; AGUIAR, E.C. O Impacto do Tamanho da Classe na Percepção de Qualidade de Alunos de uma Instituição de Ensino Superior Privada Brasileira. Revista Gestão e Planejamento, v. 19, p. 191-207, 2018.

PHAM, H-H; LAI, S.L. Higher education as an extended duration service: an investigation of the determinants of Vietnamese overseas student loyalty. Journal of Studies in International Education, v. 20, n. 5, p. 454-471, 2016.

POFFO, G.D.; MARINHO, S.V. Qualidade na percepção discente do curso de administração. Revista Gestão Universitária na América Latina, v. 6, n. 2, p. 210-230, 2013.

PRAKASH, G. Understanding service quality: insights from the literature. Journal of Advances in Management Research, v. 16, n. 1, p. 64-90, 2019.

SAPELLI, C.; ILLANES, G. Class size and teacher effects in higher education. Economics of Education Review, v. 52, p. 19-28, 2016.

Sindicato das Mantenedoras de Ensino Superior. Mapa do ensino superior no Brasil 2016. São Paulo, Convergência, 2016.

SORESCU, A.; SORESCU, S.M. Customer satisfaction and long-term stock returns. Journal of Marketing, v. 80, p. 110-115, 2016.

SPATHIS, C.; PETRIDOU, E.; GLAVELI, N. Managing Service Quality in Banks: customer's gender effects. Managing Service Quality, v. 14, p. 90-102, 2004.

SUMAEDI, S.; MAHATMA, G.; BAKTI, Y.; METASARI, N. An empirical study of state university students' perceived service quality. Quality Assurance in Education, v. 20, p. 164-183, 2012.

TEEROOVENGADUM, V.; NUNKOO, R.; GRÖNROOS, C.; KAMALANABHAN, T.J.; SEEBALUCK, A.K. Higher education service quality, student satisfaction and loyalty. Validating the HESQUAL scale and testing an improved structural model. Quality Assurance in Education, v. 27, n. 4, p. 427-445, 2019.

TOMASSINI, R.; AQUINO, R.; CARVALHO, F.A. Expectativas e percepções em sequencias de serviços. RAE Eletrônica, v. 7, n. 2, 2008.

VIEIRA-SANTOS, J.; HENKLAIN, M.H.O. Contingências sociais que dificultam o engajamento do professor universitário em relações de qualidade com seus alunos. Revista Perspectivas, v. 8, n. 2, p. 200-214, 2017.

WEBB, O.J.; COTTON, D.R.E. Deciphering the sophomore slump: changes to student perceptions during the undergraduate journey. Higher Education, v. 77, p. 173-190, 2019. 
WESTERLUND, J. Class size and student evaluations in Sweden. Education Economics, v. 16, n. 1, p. 19-28, 2008.

WITZE, A. Universities will never be the same after the coronavirus crisis. Nature, v. 582, p. 162-164, 2020.

WRIGHT, M.C.; BERGOM, I.; BARTHOLOMEW, T. Decreased class size, increased active learning? Active Learning in Higher Education, v. 20, n. 1, p. 51-62, 2019.

YE, J.; DONG, B.; LEE, J-Y. The long-term impact of service empathy and responsiveness on customer satisfaction and profitability: a longitudinal investigation in a healthcare context. Marketing Letters, v. 28, p. 551-564, 2017.

ZEITHAML, V.A. Consumer Perception of Price, Quality, and Value: A Means-End Model and Synthesis of Evidence. Journal of Marketing, v. 52, n. 3, p. 2-22, 1988.

ZEITHAML, V.A.; PARASURAMAN, A.; BERRY, L.L. Delivering Quality Service: Balancing Customer Perceptions and Expectations. New York, The Free Press, 1990. 